\title{
Mikania micrantha Extract Ameliorates Hypercholesterolemia by Inhibiting HMG-CoA Reductase and Acetyl-CoA Acetyltransferase 2 in High Cholesterol-Fed Rats
}

\author{
Azlinda Ibrahim, Nurul Husna Shafie *, Norhaizan Mohd Esa, Siti Raihanah Shafie and Hasnah \\ Bahari
}

\begin{abstract}
The present study was aimed to determine the effect of ethyl acetate of Mikania micrantha stems (EAMMS) extract in hypercholesterolemia-induced rats. Rats were divided into a normal group (NC) and hypercholesterolemia induced groups; hypercholesterolemia control group (PC), Simvastatin group (SV) $(10 \mathrm{mg} / \mathrm{kg})$ and EAMMS extract groups at different dosages of 50, 100 and $200 \mathrm{mg} / \mathrm{kg}$, respectively. Blood serum and tissues were collected for biochemical and enzyme analysis. Total cholesterol (TC), triglycerides (TG), low-density lipoprotein cholesterol (LDL-C), high-density lipoprotein cholesterol (HDL-C), aspartate aminotransferase (AST), alanine aminotransferase (ALT), urea, creatinine, as well as enzymes of HMG-CoA reductase (HMGCR) and acetyl-CoA acetyltransferase 2 (ACAT2) levels, were measured. Feeding rats with high cholesterol diet for eight weeks resulted in a significantly $(p<0.05)$ increased of TC, TG, LDL-C, AST and ALT levels. Meanwhile, the administration of EAMMS extract (50, 100 and $200 \mathrm{mg} / \mathrm{kg}$ ) and simvastatin $(10 \mathrm{mg} / \mathrm{kg})$ significantly reduced $(\mathrm{p}<0.05)$ the levels of $\mathrm{TC}, \mathrm{TG}$ and LDL-C compared to rats in the PC group. Furthermore, all EAMMS and SV-treated groups showed a higher HDL-C level compared to both NC and PC groups. No significant difference was found in the level of ALT, AST, urea and creatinine between the different dosages in EAMMS extracts. Treatment with EAMMS also exhibited the highest inhibition activity of enzyme HMGCR and ACAT2 as compared to the control group. In conclusion, these findings demonstrated that EAMMS extract had antihypercholesterolemia properties and could be used as an alternative treatment for hypercholesterolemia.
\end{abstract}

Keywords: Mikania micrantha; anti-hypercholesterolemia; lipid profile; HMG-CoA reductase; ACAT2; neutraceuticals 